\title{
New research advancement in electrical engineering field of China-Reviews on special issue of Science in China Series E: Technological Sciences
}

\author{
MEI ShengWei ${ }^{1 \dagger} \&$ MA Jin ${ }^{2}$ \\ ${ }^{1}$ State Key Laboratory of Power System, Department of Electrical Engineering, Tsinghua University, Beijing 100084, China; \\ ${ }^{2}$ Key Laboratory of Power System Protection and Dynamic Security Monitoring and Control of Ministry of Education, North China \\ Electric Power University, Beijing 102206, China
}

Based on, but not limited to the research results of electrical engineering published in the special issue of Science in China Series E, June 2008, this essay gives a brief review on a wide range of state-of-art advancement in electrical engineering field of China, which includes power system modeling, analysis and control, risk assessments in power system, etc.

electrical engineering, new research advancement

With the fast development of economy, China's power industry is experiencing a golden period. The installed generation capacity in China is increasing by $30000 \mathrm{MW}$ to $40000 \mathrm{MW}$ per year. The construction of $1000 \mathrm{kV}$ AC and $\pm 800 \mathrm{kV}$ DC ultra-high transmission system is undergoing. The Three Gorges Hydro Power Station has been in operation. Regional power grids in China are interconnected, transferring electrical energy to the areas with high demands, which, in turn, boost the national economy. China's power grid is becoming one of the most complex power systems in the world. The interconnected power system brings both opportunities and new challenges to system operation and control. The experiences in operating such a big and complex system are still lacking in the whole world. On August 14, 2003, the interconnected North American power grid collapsed after several initial contingencies. The blackout covered an area of 24000 square kilometers. More than 100 generators including 22 nuclear generators were tripped. $61800 \mathrm{MW}$ loads were lost and the affected population reached 50000000. To prevent the similar tragedy in China, Chinese academic researchers and engineers spare no efforts in finding new operation mechanisms, developing novel theories and practicing the most advanced technologies in China's power industry.

The target of the Chinese power industry focuses on improving the reliability of a large power grid and promoting sustainable developments. To reach such a target, many aspects have to be addressed and a great variety of fundamental researches have to be done. The electromagnetic compatibility (EMC) is one important issue. Owing to the high-power ratings and high-switching frequencies, the rate of changing in voltages and currents has become very large, which results in severe electromagnetic interference (EMI) noise. Ma et al. ${ }^{[1]}$ propose a noise source lumped circuit modeling and identification method, which includes an EMI modeling method based on multiple slope approximation of switching transients and double Fourier integral method modeling PWM conversion units. A series of EMI modeling and analysis methods that can be applied to the accurate prediction of EMI noise in the entire conducted EMI range has been developed. Their work has laid down an academic foundation for the EMC studies of power electronic systems.

The reliability of the power system seeks for an equilibrium balancing the economy and the security. Therefore, the risk assessment is necessary for power system operation, both in the steady state and in the dynamic state. Yu et al. ${ }^{[2]}$

Received August 18, 2008; accepted January 13, 2009 doi: 10.1007/s11434-009-0145-z

†Corresponding author (email: meishengwei@mail.tsinghua.edu.cn)

Supported by the National Science Fundation of China (Grant No. 50525721, 50595411), and the National Basic Research Program of China (Grant No. 2004CB217900) 
propose a practical dynamic security region (PDSR) based dynamic security risk assessment and optimization model. The cost of comprehensive security control and the influence of uncertainties of power injections are considered in the model of dynamic security risk assessment. An optimal solution is obtained by optimizing preventive and emergency costs and contingency set decomposition. Also contributing to the dynamic security region (DSR), Xue et al. ${ }^{[3]}$ propose a new transient stability margin based on a new expression of DSR, which has been tested numerically in the 10-generator 39-bus New England test system. Risk evaluation has also been applied to power system operation scheduling. Kang et al. ${ }^{[4]}$ propose the multidimensional sequence operation theory (MSOT) as a development of the basic single dimension sequence operation theory (SOT). Application of this theory to power system generation scheduling shows its practical value in the power industry.

To meet the demand of high stability, high quality, and low losses of power systems, Lu et al. ${ }^{[5]}$ establish the advanced energy management system (AEMS) on the foundation of the hybrid automatic control theory and the "event-driven" strategy. AEMS is novel from theory to engineering applications. The first AEMS has been operating in Shanghai power system for one and half a year. Due to the limitation of the mathematical tool, the power system has been initially researched as a linear model. There has been some research in nonlinear dynamics of power systems ${ }^{[6]}$. However, the application of nonlinear control theory to power system is a big contribution of Chinese scientists to the power engineering world. The conditions to exactly linearize power system model have been proposed and tested, which open a new field for controller design in the nonlinear domain. The theoretic research results have been successfully applied to power industry. The field experiments of NR-PSS (Nonlinear Robust Power System Stabilizer) were carried out on a $300 \mathrm{MW}$ generator in Baishan Hydro Plant

1 Ma W M, Zhao Z H, Meng J, et al. Precise methods for conducted EMI modeling, analysis, and prediction. Sci China Ser E Tech Sci, 2008, 51(6): 641-655

2 Yu Y X, Wang D T. Dynamic security risk assessment and optimization of power transmission system. Sci China Ser E Tech Sci, 2008, 51(6): $713-723$

3 Xue A C, Mei S W. A new transient stability margin based on dynamic security region and its applications. Sci China Ser E Tech Sci, 2008, 51(6): $751-760$

4 Kang C Q, Yang G F, Xia Q. Development of multidimensional sequence operation theory with applications to risk evaluation in power system generation scheduling. Sci China Ser E Tech Sci, 2008, 51(6): $724-734$

5 Lu Q, He G Y, Mei S W, et al. Advanced EMS and its trial operation in shanghai power system. Sci China Ser E-Tech Sci, 2008, 51(2): $220-224$

6 Lai D W, Wang Z X. An application of bifurcation of periodic solu- of Northeast China Power Grid ${ }^{[7]}$. Field experiments, as well as large disturbance experiments on real time digital simulator (RTDS) ${ }^{[8]}$, show that the transient performance of generator excitation system equipped with NR-PSS are dramatically improved compared with the traditional PSS. Based on the nonlinear robust control theory and the exactly linearized generator modes of multi-machine power systems, the design principles of NR-PSS have been proposed and developed successfully by Lu et al.

Modeling is another important issue in power system, and is also the basis for power system analysis and control. Wang et al. ${ }^{[9]}$ build the model of dual-stator winding multi-phase high-speed induction generator with rectifier load and propose a new method to divide the whole system into two sub-systems. Then the circuit method and the field finite element method are adopted to analyze the two sub-systems. Wu et al. ${ }^{[10]}$ dig into the basics of the control model of electronics technology and analyze in detail the essential concepts and control technologies in the renewable energy generation, which are all very important issues in the applications of wind energy and solar energy. Relay is another important component in power system, and it builds the first defense line on power system security. Many recorded blackouts are initiated by the mis-operations of the relays. Dong et al. ${ }^{[11]}$ propose an assembled fault location method for a transmission line based on single-terminal electrical quantities. The fault zone was firstly determined by impedance method with robustness; then the accurate fault position was pinpointed by travelling wave method.

Opportunities and challenges coexist in the power industry of China. Chinese researchers and engineers shoulder great responsibilities to promote new technologies and make contributions to the sustainable developments of this booming industry. More advancement in the electrical engineering field of China is sure to come!

tions to ferroresonance over-voltage in power systems. Chin Sci Bull, 1991, 36(19): 1601-1601

7 Lu Q, Mei S W, Zheng S M. Field experiments of NR-PSS for large synchronous generators on a $300 \mathrm{MW}$ machine in Baishan Hydro Plant. Sci China Ser E Tech Sci, 2007, 50(4): 516-520

8 Lu Q, Zheng S M, Mei S W, et al. NR-PSS (Nonlinear Robust Power System Stabilizer) for large synchronous generators and its large disturbance experiments on real time digital simulator. Sci China Ser E Tech Sci, 2008, 51(4): 337-352

9 Wang X H, Wu X Z. Research on dual-stator winding multi-phase high-speed induction generator with rectifier load. Sci China Ser E Tech Sci, 2008, 51(6): 674-682

$10 \mathrm{Wu}$ J, Chen S Z, Liu D. Control and power electronics technology in renewable energy, Sci China Ser E Tech Sci, 2008, 51(6): 702-712

11 Dong X Z, Shi S X, Cui T, et al. Optimizing solution of fault location using single terminal quantities. Sci China Ser E Tech Sci, 2008, 51(6): $761-772$ 